\title{
Polyp Genetics
}

\author{
Coen Laurens Klos, MD ${ }^{1}$ Sekhar Dharmarajan, MD ${ }^{1}$ \\ 1 Section of Colon and Rectal Surgery, Department of Surgery, \\ Washington University School of Medicine, St. Louis, Missouri \\ Address for correspondence Sekhar Dharmarajan, MD, Campus Box \\ 8109, 660 South Euclid Avenue, St. Louis, MO 63110 \\ (e-mail: dharmarajans@wudosis.wustl.edu).
}

Clin Colon Rectal Surg 2016;29:289-295.

\begin{abstract}
Keywords

- polyps

- genetics

- colorectal

- neoplasm
\end{abstract}

Our fundamental understanding of the pathogenesis of colorectal cancer (CRC) is derived from the elegant and classic report of Vogelstein and Fearon. ${ }^{1}$ In this model, acquired genetic mutations leading to increased function of oncogenes or loss of function in tumor suppressor genes provide a selective growth advantage to clones of cells. ${ }^{2}$ Expansion of these clones of cells initiates the transformation of normal colonic epithelium into benign neoplasms or polyps, and the accumulation of further genetic changes drives their progression to larger, more advanced neoplasms, and finally to invasive cancer, the so-called "polyp to cancer progression

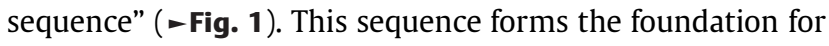
many current clinical practices regarding the screening, surveillance, and prevention of CRC. For example, the time interval between initial polyp formation and progression into cancer allows for colonoscopic screening and polypectomy, which reduces the risk of CRC. ${ }^{3-13}$ Since their original description, there have been tremendous advances in our knowledge of the genetic changes involved in the CRC, and these have led to numerous revisions of the model. Our current understanding of the polyp to cancer sequence is that it is heterogeneous and involves multiple genetic pathways, and that specific genetic alterations lead to biologically and clinically distinct subsets of CRC. Here, we review the most common types of polyps of the colon and rectum and discuss the current state of knowledge regarding the genetic pathways by which these polyps develop into cancer.

\section{Adenomatous Polyps}

Adenomatous polyps are the most common dysplastic sporadic polyps of the colon and rectum and the most important precursor lesion to CRC. ${ }^{14}$ The important risk factors for the development of adenomatous polyps include advanced age, smoking status, and moderate-to-high consumption of alcohol. ${ }^{15}$ In the United States, $25 \%$ of people by the age of 50 , and $50 \%$ by the age of 70 , will develop adenomatous polyps of the colon and rectum. ${ }^{16}$ Endoscopically, adenomas can be classified as pedunculated, sessile, flat, or depressed. Microscopically, these polyps are classified based on their glandular
Issue Theme Colorectal Polyps and Polyposis Syndromes; Guest Editor: Paul E. Wise, MD, FACS, FASCRS
Copyright $\odot 2016$ by Thieme Medical Publishers, Inc., 333 Seventh Avenue, New York, NY 10001, USA. Tel: +1(212) 584-4662.
DOI http://dx.doi.org/ 10.1055/s-0036-1582442. ISSN 1531-0043. 


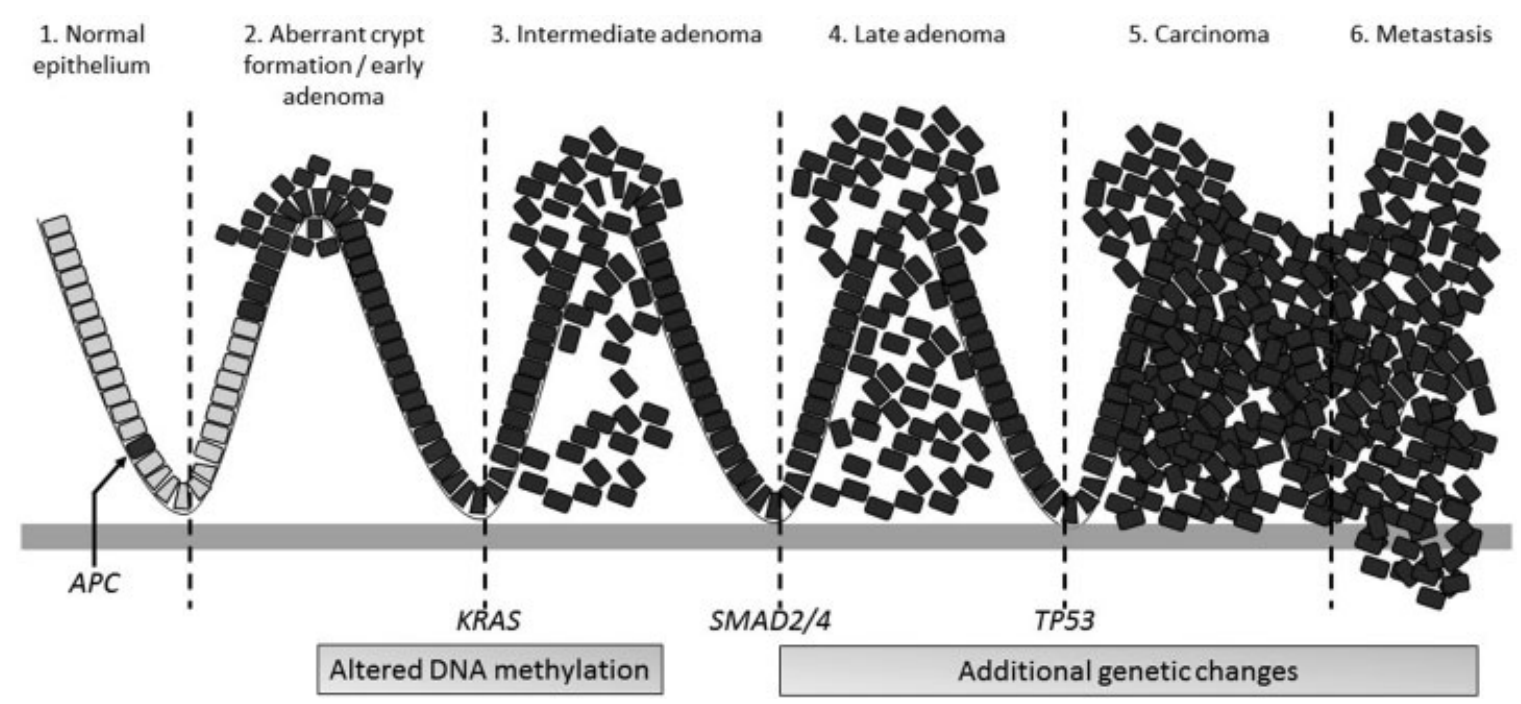

Fig. 1 Diagram of the adenoma to carcinoma sequence starting with: (1) Normal epithelium, an inactivation mutation occurs in the APC gene. (2) Inactivation of APC leads to translocation of $\beta$ catenin to the cell nucleus, causing an increase in cell proliferation resulting in aberrant crypt formation. (3-5) Due to altered DNA methylation, additional gene mutations accumulate including KRAS early on, SMAD2/4, and eventually TP53. (6) As the number of genetic changes continues to increase, cells develop the ability to migrate and metastasize.

structure: tubular, villous, or tubulovillous. Up to $80 \%$ of this subset of adenomas is tubular. The remaining share is evenly distributed between villous and tubulovillous adenomas. Size and underlying histology are the most important factors in determining the malignant potential of adenomatous polyps. Villous adenomas have the strongest association with progression to cancer. When the size of the polyp extends beyond $2 \mathrm{~cm}$, the risk of cancer within the lesion is as much as 50\%.

\section{Adenomatous Polyps and Chromosomal Instability}

The genetic pathway by which adenomatous polyps develop into cancer, as initially described by Fearon and Vogelstein, is responsible for the majority of sporadic CRC (range, 70$80 \%) .{ }^{17}$ In this model, inactivating mutations in the adenomatous polyposis coli (APC) tumor suppressor gene located on chromosome $5 q$ are the initiating genetic events, giving rise to the first identifiable histologic lesion implicated in CRC formation, the aberrant crypt focus. ${ }^{18}$ Inactivation of APC leads to upregulation of Wingless/Wnt $\beta$-catenin signaling, displacing $\beta$-catenin to the nucleus, where it activates cell proliferation factors such as CMYC and cyclin D1. This highlights the role of the APC gene as a gatekeeper gene in normal colorectal epithelium, responsible for maintaining constant cell numbers in renewing cell populations, with mutations in $A P C$ resulting in a permanent imbalance favoring cell division over cell death. ${ }^{19}$

Subsequent to a mutation in APC or other genes of the Wnt signaling pathway, other early genetic events that foster the progression from early-to-intermediate adenoma are KRAS or p53 mutations. ${ }^{2,3}$ KRAS is an oncogene that is a member of the downstream signaling pathway of the epidermal growth factor receptor (EGFR). It signals through $B R A F$ to activate the mitogen-activated protein (MAP) kinase pathway, inducing cell proliferation and inhibiting apoptosis. Mutations in KRAS are seen in up to $50 \%$ of CRC, and identification of KRAS mutations in the adenoma to carcinoma sequence is a prime example of how advances in our understanding of polyp genetics influence the clinical treatment of patients with CRC. $^{2,3}$ The recognition that activation of EGFR signaling is common in CRC has led to the development of monoclonal antibodies to EGFR that have shown moderate efficacy in improving survival in patients with metastatic CRC. ${ }^{20,21}$ As KRAS is the downstream target of EGFR, it was recognized that treatment with EGFR antibodies may not be effective in patients with KRAS mutations, and this has been borne out in several studies. ${ }^{20,22}$ This observation has allowed the targeted use of this expensive agent only in patients most likely to benefit from this therapy. Other genetic events related to KRAS mutations are mutations in the phosphatidylinositol 3-kinase (PI3K) pathway, another protein kinase pathway important in cell growth, proliferation, and survival. The net result of these genetic changes is DNA hypomethylation and chromosomal instability (CIN) or loss of heterozygosity through defects in genes that regulate the formation of the mitotic spindle and the proper alignment and segregation of chromosomes during mitosis. This CIN or aneuploidy most commonly results in the loss of chromosome $17 \mathrm{p}$ and 18 . This causes inactivation of deleted in colon cancer $(D C C)$ gene on chromosome 18q, which encodes an adhesion molecule that facilitates apoptosis and therefore tumor suppression. This is thought to be an important step in the transition from intermediate-to-advanced adenoma. Another genetic pathway altered by the loss of chromosome $18 \mathrm{q}$ is the transforming growth factor- $\beta$ cell signaling pathway through the loss of SMAD2 and SMAD4. The final genetic event thought to be important in the development of invasive cancer is the loss of chromosome $17 \mathrm{p}$ and the $p 53$ gene. The p53 protein functions as a key transcriptional regulator of genes important in cell cycle checkpoints, apoptosis, and angiogenesis. While not all of these genes are mutated in every CRC, the 
selective pressures that lead to the clonal evolution and growth advantage of polyps are largely the same across all CRCs. Thus, the aforementioned genetic changes of the canonical adenoma to carcinoma pathway are responsible for 70 to $80 \%$ of all sporadic CRC.

\section{Adenomatous Polyps and Microsatellite Instability}

The first evidence that there may be multiple pathways leading to CRC came from genetic analysis of CRC in patients with Lynch syndrome, whose tumors had a distinct histologic appearance and genetic signature when compared with sporadic tumors of the CIN pathway. Lynch syndrome, the most common hereditary CRC syndrome, is an autosomaldominant condition that accounts for 3 to $5 \%$ of all CRC. ${ }^{23}$ Histologically, Lynch syndrome CRC differs from sporadic CRC of the CIN pathway in that it is more commonly right sided, more often poorly differentiated with mucinous features, and has a marked peritumoral lymphocytic infiltrate. While the adenomatous polyp is thought to be the precursor of CRC in Lynch syndrome, the progression through the polyp to cancer sequence is thought to be much more rapid. Despite the adverse histologic features seen in Lynch syndrome CRC, however, is generally associated with a better prognosis than sporadic CRC of the CIN pathway. ${ }^{24}$

From a genetic perspective, Lynch syndrome CRC does not display CIN and is typically diploid. The characteristic genetic feature of Lynch-associated CRC is the expansion and contraction of nucleotide repeats in DNA, a pattern termed microsatellite instability (MSI), which is not a feature of the CRC of the CIN pathway. In Lynch syndrome, this MSI is due to inactivation of one of several DNA mismatch repair genes (MLH1, MSH2, MSH6, PMS2) that are responsible for its inheritance and form the basis of genetic testing for this syndrome. In another clinical application of genetic advances, testing was developed for MSI by two separate methods. The first method involves genetic analysis of a panel of microsatellite sequences in DNA in normal mucosa as well as tumor to detect expansion or contraction of microsatellites. The second is to perform immunohistochemical analysis of tumor tissue for expression of the four DNA mismatch repair gene products, as loss of one or more of these is indicative of MSI. Thus, we see the emergence of two distinct subsets of CRC with different clinical features and molecular pathogenesis: the CIN pathway, accounting for 70 to $80 \%$ of CRC and characterized by CIN and microsatellite stability (MSS); and, CRC associated with Lynch syndrome, accounting for 3 to $5 \%$ of CRC and characterized by chromosomal stability and MSI. After testing for MSI became more widespread, it became readily apparent that there was a subset of sporadic CRC that displayed MSI in which there was no detectable history of inheritance and no detectable mutation in DNA mismatch repair genes. These observations led to the establishment of a third genetic pathway to CRC, termed the DNA hypermethylation pathway (see below).

\section{Serrated Polyps}

Hyperplastic polyps, a type of "serrated polyp," were initially considered the most common nonneoplastic polyp of the colon and rectum. ${ }^{25}$ The most important risk factor for the development of hyperplastic polyps is smoking. ${ }^{26}$ Unlike adenomatous polyps, as described above, hyperplastic polyp incidence does not significantly increase with age. ${ }^{27}$ Characterized by a serrated pattern of incorporated crypts, these polyps belong to the family of serrated polyps, consisting of hyperplastic polyps, sessile serrated adenomas/polyps (SSA/ $\mathrm{P}$ ), and traditional serrated adenomas (TSA) (although pathologists still debate the semantics of these polyps). Hyperplastic polyps can be subclassified by histologic appearance into microvesicular (MVHP), goblet cell (GCHP), and the least common mucin poor variants. ${ }^{28-30}$ There is now evidence that these hyperplastic polyps are likely to be precursor lesions to SSA/Ps or TSAs that do have the potential to evolve into cancer through a third genetic pathway to the CRC, the DNA hypermethylation pathway.

\section{Serrated Polyps and DNA Hypermethylation}

Recognition of a subset of CRCs that displayed MSI but did not have detectable mutations in DNA mismatch repair genes led to further genetic analysis of this subset. This revealed inactivation of the DNA mismatch repair gene $M L H 1$, not through direct mutation, but rather by hypermethylation of the promoter region of the gene. This increased DNA hypermethylation is not just confined to the promoter region of MLH1, but also over promoter areas rich in cytosine and guanine dinucleotide repeats for a large number of genes, a process termed the $\mathrm{CpG}$-island methylator phenotype (CIMP). CRCs arising from this pathway are similar to those in Lynch syndrome in that they do not display CIN but do display MSI. These cancers also display a unique clinical phenotype in that they tend to occur more commonly in the right colon, have a predilection for older females, and carry a better prognosis than CIN pathway CRCs. Although somewhat controversial, some studies also suggest that sporadic MSI CRC may not respond to 5-FU based chemotherapy regimens as well as CIN CRC. ${ }^{25}$

While the DNA hypermethylation pathway is genetically fundamentally distinct from the CIN pathway in that it is driven primarily by abnormalities in regulation of gene methylation as opposed to mutational events, an initial genetic mutation similar to the APC mutation in the CIN pathway is thought to be the inciting event in the transformation of normal colonic epithelium into a premalignant polyp. It is believed that a mutation in BRAF is the initial event driving the conversion of normal mucosa into an MVHP or SSA/P. From here, the BRAF mutation leads to activation of the MAP kinase pathway and induces DNA hypermethylation or CIMP. SSA/Ps can therefore be marked as CIMP high. This cascade of events leads to silencing of upstream Wnt pathway mediators leading to displacement of $\beta$-catenin to the nucleus. In addition, in the SSA/P specifically, hypermethylation of mismatch repair gene $M L H 1$ results in a high level of MSI. Hypermethylation of MLH1 is found in 36\% of MCHP, 70\% of SSA/Ps, and close to $90 \%$ of sporadic MSI CRC. ${ }^{31}$ The rate of progression through this sequence is variable, but it is thought that once MSI occurs, there is a more rapid progression to cancer. Accumulation of other epigenetic events such 
as the silencing of tumor suppressor gene p16 facilitates further deterioration into carcinoma. ${ }^{18}$ Thus, CRC arising from the DNA hypermethylation pathway is often termed as MSI high and CIMP high.

The identification of a BRAF mutation as the initial event in the DNA hypermethylation pathway is important for three reasons. First, it establishes the hyperplastic or serrated polyp as the precursor lesion of sporadic MSI CRC, as BRAF mutations are found in 75 to $80 \%$ of MVHPs or SSA/Ps, but rarely, if ever, in traditional adenomas. ${ }^{31}$ Second, it provides a method to classify CRC according to the genetic pathway through which it develops. In this algorithm, immunohistochemical analysis of DNA mismatch repair proteins is performed in tumor tissue. If they are preserved, the cancer is likely from the CIN pathway. If there is loss of expression of $\mathrm{MSH} 2, \mathrm{MSH}$, or PMS2, then the tumor is more likely a Lynch syndrome CRC. If there is loss of expression of MLH1 ( \pm loss of PMS2 as well) then BRAF mutation testing is performed to determine if the tumor is a Lynch syndrome CRC (BRAF-mutation negative) or a sporadic MSI CRC associated with the DNA hypermethylation pathway (BRAF-mutation positive). Finally, since BRAF is downstream of KRAS, it is also true that BRAF-mutated CRC is not responsive to therapy with EGFR antibodies.

More recently, there is evidence to support the existence of an alternate genetic pathway for the transformation of serrated polyps to CRC. In this pathway, KRAS mutation is believed to be the initial event in the transformation of normal colonic epithelium into GCHPs or TSAs. This mutation does not induce much DNA hypermethylation, and subsequently these tumors are characterized as CIMP low and are MSS as there is no loss of MLH1 activity. This pathway has been associated with hypermethylation of the DNA repair gene, 0-6-methylguanine-DNA-methytransferase (MGMT). ${ }^{31}$ The activity of $p 16$ is initially upregulated in this situation, possibly in an attempt to block, uncontrolled cell proliferation initiated by dysregulation of the Wnt $\beta$-catenin signaling pathway. Eventually, methylation of MGMT and other not well-known targets, in combination with early suppression of p53, results in CRC that is characterized as CIMP low and MSS and is associated with an aggressive phenotype. -Fig. 2 summarizes the main genetic changes associated with the different subtypes of CRC.

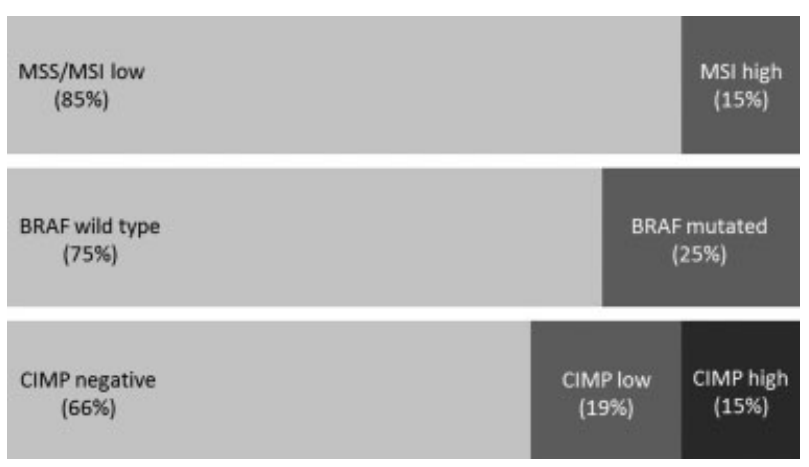

Fig. 2 Each bar reflects the distribution of a specific molecular characteristic among colorectal cancers. CIMP, CpG-island methylator phenotype; MSI, microsatellite instability; MSS, microsatellite stable.

\section{Adenomatous Polyposis Syndromes}

Adenomatous polyposis syndromes (addressed in more detail in article "Adenomatous Polyposis Syndromes: Diagnosis and Management" by Mitchem and Hall on pp. 321-329) constitute the etiology of a minority of all CRC ( $\sim 1 \%$ or less). These syndromes are caused by germline mutations in genes that cause a predisposition to the formation of adenomatous polyps. The most well-known is familial adenomatous polyposis, which is an autosomal-dominant syndrome characterized by the development of hundreds of adenomas in the second and third decade of life. This syndrome is caused by germline mutations in the APC gene and follows the canonical adenoma-carcinoma pathway as outlined above. The phenotype of the syndrome is governed by the mutation site in the APC gene, with mutations at either end of the gene or in exon nine associated with a milder phenotype. ${ }^{32}$ MYH-polyposis, or MAP, is an autosomal recessive syndrome that causes the development of multiple colorectal adenomas (usually $<100$ ) in the third and fourth decades of life. This syndrome is due to germline mutations in the MUTYH gene, a base excision repair gene involved in the repair of genetic mutations caused by reactive oxygen species and DNA damage due to methylation, deamination, and hydroxylation. Germline mutations in POLE and POLD1 genes have been identified in families characterized by members with multiple adenomas and early-onset CRC. ${ }^{32}$ These genes encode proteins important in leading and lagging strand synthesis during DNA replication as well as proteins involved in DNA proofreading to ensure fidelity during DNA replication. Again, these syndromes are addressed in more detail in article "Adenomatous Polyposis Syndromes: Diagnosis and Management" by Mitchem and Hall on pp. 321-329.

\section{Hamartomatous Polyps}

Hamartomatous polyps represent developmental malformations wherein the epithelial surface and underlying lamina propria are arranged abnormally, but there are no premalignant changes. Hamartomatous polyps can generally be divided into two types: juvenile (or retention polyps) and PeutzJeghers type polyps. In their sporadic form, hamartomatous polyps are not associated with an increased risk of malignancy. There are hamartomatous polyposis syndromes, however, in which germline mutations lead to formation of multiple polyps with an associated increased risk of malignancy. These are also addressed in article "Hamartomatous Polyps and Associated Syndromes" by Cone.

\section{Juvenile Polyps}

The term juvenile polyp refers to polyp histology rather than their age of presentation since they can occur at any age. Histologically, juvenile polyps are characterized by mucusfilled, cystically dilated glands and an abundant and expanded lamina propria that is usually infiltrated by inflammatory cells, often eosinophils. Beginning in childhood, patients with juvenile polyposis syndrome (JPS) develop multiple hamartomatous polyps throughout the gastrointestinal (GI) tract, especially in the colon and stomach, with CRC risk of $60 \%$ by 
age 60 and increased risk of gastric cancer as well. ${ }^{33}$ Most patients (75\%) presenting with suspected JPS have a parent with the syndrome. ${ }^{33}$ The underlying germline mutations that are causative occur in the BMPR1A and SMAD4 tumor suppressor genes, which together account for approximately $60 \%$ of all JPS patients. ${ }^{33}$ There is evidence to suggest that patients with a SMAD4 mutation have a more aggressive phenotype with higher frequency of gastric polyps and cancer compared with patients with BMPR1A mutations. ${ }^{33}$ Hereditary hemorrhagic telangiectasia (or Osler-Weber-Rendu syndrome) may also develop in patients with SMAD4 mutations.

BMPR1A and SMAD4 are both important components of the TGF- $\beta /$ BMP signaling pathway, which regulates cell proliferation, differentiation, and apoptosis. ${ }^{34,35}$ The protein BMPR1A is a transmembrane receptor kinase that when phosphorylated transduces the signal by phosphorylating SMAD2 and 3, or SMAD1, 5, and 8. These will form a complex with the common mediator, SMAD4, and translocate to the nucleus where it will regulate the above-mentioned process- es. Unregulated proliferation, differentiation, and apoptosis lead to activating mutations of oncogenes and silencing of, or mutations in, tumor suppressor genes leading to subsequent development of cancer.

\section{Peutz-Jeghers Polyps}

Peutz-Jeghers polyps are commonly found in the small bowel and colon and are characterized by cystic gland dilation and arborizing smooth muscle. Peutz-Jeghers syndrome (PJS) is an autosomal-dominant condition typified by the presence of Peutz-Jeghers polyps throughout the GI tract and mucocutaneous hyperpigmentation. The incidence of Peutz-Jeghers polyps in the setting of PJS is anywhere between 1:83,000 and $1: 200,000$ with $55 \%$ of patients having an affected parent. ${ }^{36} \mathrm{~A}$ germline mutation in the STK11 gene is present in $94 \%$ of patients fulfilling diagnostic criteria for PJS. STK11 is a tumor suppressor gene that when silenced by mutation causes hyperactivation of the mTOR pathway. ${ }^{36}$ This in turn leads to increased AKT signaling, stimulating cell survival, and

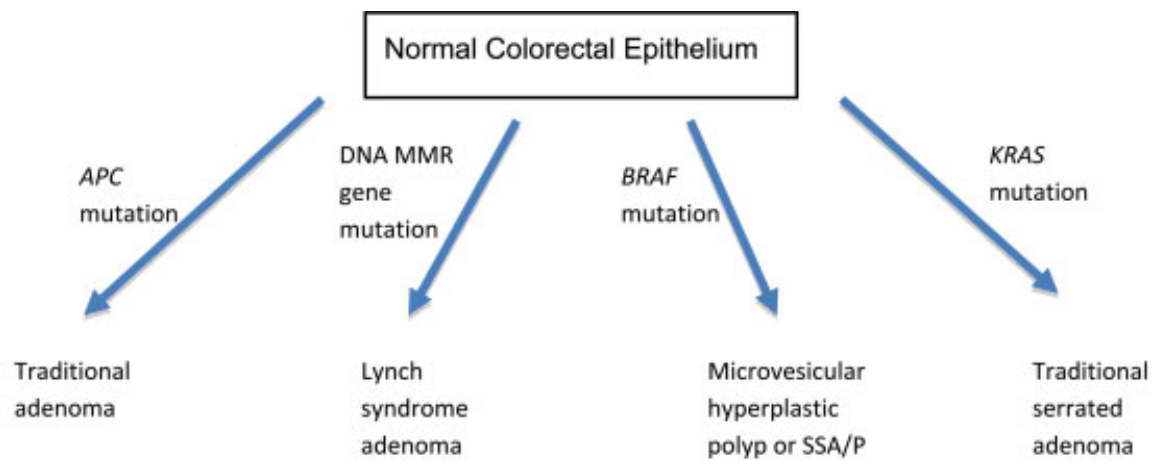

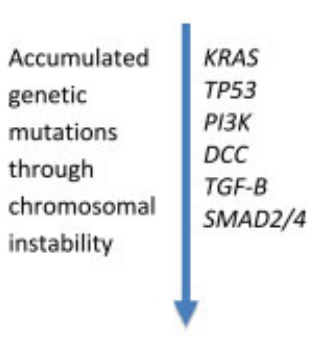

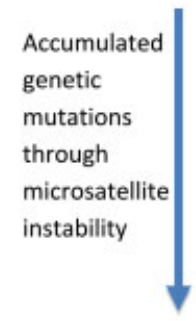

Invasive Cancer

Invasive Cancer

$\begin{array}{ll}\text { 70-80\% CRC } & 3-5 \% \text { CRC } \\ \text { Aneuploid } & \text { Diploid } \\ \text { MSS } & \text { MSI } \\ \text { CIMP-negative } & \text { CIMP-negative } \\ \text { KRAS mut } & \text { KRAS wt } \\ \text { BRAF wt } & \text { BRAF wt }\end{array}$

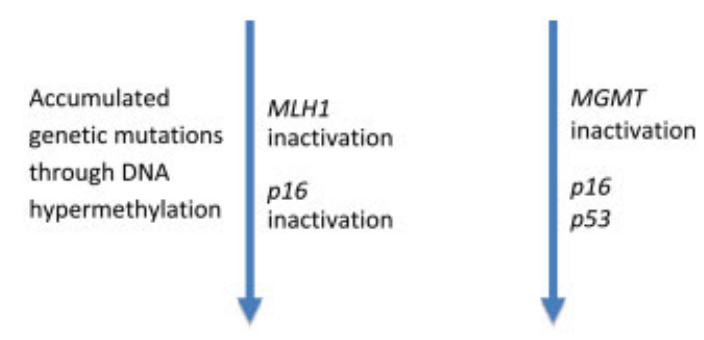

Invasive Cancer

Invasive Cancer

$\begin{array}{ll}\text { 10-15\% CRC } & \text { ? incidence } \\ \text { Diploid } & \text { Diploid } \\ \text { MSI } & \text { MSS } \\ \text { CIMP-high } & \text { CIMP-low } \\ \text { KRAS wt } & \text { KRAS mut } \\ \text { BRAF mut } & \text { BRAF wt }\end{array}$

Fig. 3 Summary of genetic pathways to colon cancer. There are four main initial gene mutations: APC, adenomatous polyposis coli; MMR, mismatch repair, BRAF, or KRAS. These mutations lead to different types of early lesions before additional genetic changes accumulate. These additional genetic changes are caused by chromosomal instability, microsatellite instability, or DNA hypermethylation respectively. Increasing numbers of gene mutations eventually lead to invasive cancers with specific histologic and molecular characteristics depending on the original mutational event. CIMP, CpG-island methylator phenotype; MSI, microsatellite instability; MSS, microsatellite stable; Mut, mutant; Wt, wild-type. 
activation of cyclin D1, which stimulates cell cycle progression. It is also an upstream regulator of AMP-activated protein kinase and as such is involved in various processes such as cell metabolism, cell polarity, apoptosis, and DNA-damage responses.

\section{PTEN Hamartoma Syndrome}

The PTEN hamartoma syndrome is an inherited multiple hamartoma syndrome associated with high risk of cancer in various organs. This syndrome is a collective term for several clinical syndromes with overlapping features, including Cowden disease, Lhermitte-Duclos disease, and Bannayan-RileyRuvalcaba syndrome. A germline mutation in the phosphatase and tensin homolog (PTEN) tumor suppressor gene is responsible for these syndromes. PTEN is a tumor suppressor gene coding for a protein that downregulates the phosphatidylinositol 3-kinase (PI3K), AKT, and mTOR pathways. ${ }^{37,38}$ In addition, PTEN has specific function in the cell nucleus, including regulation of the formation and function of APC$\mathrm{CDH} 1{ }^{39}$ Together, these changes lead to an increased cell survival and cell proliferation through cell cycle progression.

\section{Conclusion}

As evidenced from the above discussion, our understanding of the genetic events involved in the polyp-to-cancer sequence has evolved greatly since its original conception by Vogelstein and Fearon. The current models would suggest that there are at least three distinct genetic pathways that drive the adenoma to carcinoma sequence, leading to different subsets of CRCs that can be classified according to genotype and phenotype (-Fig. 3). The CIN pathway is driven largely by mutations in oncogenes and tumor suppressor genes, with an $A P C$ mutation postulated as the inciting event, and accounting for the majority of all CRC. CRC that results from this pathway is a KRAS mutation positive, displays aneuploidy or CIN, and is CIMP-negative and MSS. The MSI pathway is incited by germline mutations in DNA mismatch repair genes and cause the Lynch syndrome, which accounts for 3 to $5 \%$ of all CRC. CRC associated with Lynch syndrome is KRAS/BRAF-mutation negative, shows MSI, is diploid with no CIN, and CIMP negative. Finally, the DNA hypermethylation pathway results from alterations in methylation patterns of genes rather than gene mutations themselves. This pathway accounts for 10 to $15 \%$ of all CRC and is characterized by chromosomal stability, MSI, BRAF-mutation positive, and CIMP high. Our understanding of this pathway has led to the recognition of hyperplastic and serrated polyps as an important precursor of CRC. The discovery of different genetic pathways of CRC has had a significant effect on clinical algorithms for the diagnosis and treatment of CRC. Genetic testing of the CRC can lead to the identification of patients with hereditary CRC as well as change the chemotherapeutic regimens used to treat these patients.

Research focused on identifying additional genes implicated in colorectal polyp formation and progression to carcinoma is ongoing. An improved understanding of the key genetic processes involved will allow for tailored treatment strategies.
These strategies may include chemoprevention of lesions developing in patients at risk based on known germline mutations, early recognition and removal of precursor lesions, and preventative resection of lesions with a suspected aggressive phenotype based on genotype. Future investigation should be focused on developing targeted gene therapy. Currently, the genes known to be involved in the process of developing CRC are those that are implicated most commonly and/or relatively early in the cascade of events. As described, these early genetic events trigger alterations in the function of many "downstream" genes, many of which are still to be identified. It is important that all clinicians treating patients with CRC stay informed of these new developments as diagnostic and treatment strategies may be adjusted accordingly.

\section{References}

1 Vogelstein B, Fearon ER, Hamilton SR, et al. Genetic alterations during colorectal-tumor development. N Engl J Med 1988;319(9): 525-532

2 Fearon ER. Molecular genetics of colorectal cancer. Annu Rev Pathol 2011;6:479-507

3 Brenner H, Kloor M, Pox CP. Colorectal cancer. Lancet 2014; 383(9927):1490-1502

4 Bosman F, Yan P. Molecular pathology of colorectal cancer. Pol J Pathol 2014;65(4):257-266

5 Shroff J, Thosani N, Batra S, Singh H, Guha S. Reduced incidence and mortality from colorectal cancer with flexible-sigmoidoscopy screening: a meta-analysis. World J Gastroenterol 2014;20(48): 18466-18476

6 Lin OS, Kozarek RA, Cha JM. Impact of sigmoidoscopy and colonoscopy on colorectal cancer incidence and mortality: an evidence-based review of published prospective and retrospective studies. Intest Res 2014;12(4):268-274

7 Hewett DG, Rex DK. The big picture: does colonoscopy work? Gastrointest Endosc Clin N Am 2015;25(2):403-413

8 Atkin WS, Edwards R, Kralj-Hans I, et al; UK Flexible Sigmoidoscopy Trial Investigators. Once-only flexible sigmoidoscopy screening in prevention of colorectal cancer: a multicentre randomised controlled trial. Lancet 2010;375(9726):1624-1633

9 Schoen RE, Pinsky PF, Weissfeld JL, et al; PLCO Project Team. Colorectal-cancer incidence and mortality with screening flexible sigmoidoscopy. N Engl J Med 2012;366(25):2345-2357

10 Segnan N, Armaroli P, Bonelli L, et al; SCORE Working Group. Onceonly sigmoidoscopy in colorectal cancer screening: follow-up findings of the Italian Randomized Controlled Trial-SCORE. J Natl Cancer Inst 2011;103(17):1310-1322

11 Winawer SJ, Zauber AG, Ho MN, et al; The National Polyp Study Workgroup. Prevention of colorectal cancer by colonoscopic polypectomy. N Engl J Med 1993;329(27):1977-1981

12 Nishihara R, Wu K, Lochhead P, et al. Long-term colorectal-cancer incidence and mortality after lower endoscopy. N Engl J Med 2013; 369(12):1095-1105

13 Zauber AG, Winawer SJ, O’Brien MJ, et al. Colonoscopic polypectomy and long-term prevention of colorectal-cancer deaths. $\mathrm{N}$ Engl J Med 2012;366(8):687-696

14 Giacosa A, Frascio F, Munizzi F. Epidemiology of colorectal polyps. Tech Coloproctol 2004;8(Suppl 2):s243-s247

15 Heitman SJ, Ronksley PE, Hilsden RJ, Manns BJ, Rostom A, Hemmelgarn BR. Prevalence of adenomas and colorectal cancer in average risk individuals: a systematic review and meta-analysis. Clin Gastroenterol Hepatol 2009;7(12):1272-1278

16 Rex DK, Lehman GA, Ulbright TM, et al. Colonic neoplasia in asymptomatic persons with negative fecal occult blood tests: 
influence of age, gender, and family history. Am J Gastroenterol 1993;88(6):825-831

17 Fearon ER, Vogelstein B. A genetic model for colorectal tumorigenesis. Cell 1990;61(5):759-767

18 Lao VV, Grady WM. Epigenetics and colorectal cancer. Nat Rev Gastroenterol Hepatol 2011;8(12):686-700

19 Kinzler KW, Vogelstein B. Lessons from hereditary colorectal cancer. Cell 1996;87(2):159-170

20 Siena S, Sartore-Bianchi A, Di Nicolantonio F, Balfour J, Bardelli A. Biomarkers predicting clinical outcome of epidermal growth factor receptor-targeted therapy in metastatic colorectal cancer. J Natl Cancer Inst 2009;101(19):1308-1324

21 Jean GW, Shah SR. Epidermal growth factor receptor monoclonal antibodies for the treatment of metastatic colorectal cancer. Pharmacotherapy 2008;28(6):742-754

22 Jiang Y, Kimchi ET, Staveley-O'Carroll KF, Cheng H, Ajani JA. Assessment of K-ras mutation: a step toward personalized medicine for patients with colorectal cancer. Cancer 2009;115(16): 3609-3617

23 Ahnen DJ. The American College of Gastroenterology Emily Couric Lecture-the adenoma-carcinoma sequence revisited: has the era of genetic tailoring finally arrived? Am J Gastroenterol 2011; 106(2):190-198

24 Boland CR, Goel A. Microsatellite instability in colorectal cancer. Gastroenterology 2010;138(6):2073-2087.e3

25 Rotondano G, Bianco MA, Cipolletta L, Marmo R. Serrated LESions of the COlorectum (SELCO) investigators Prevalence and characteristics of serrated lesions of the colorectum in Italy: A multicentre prospective cohort study. Dig Liver Dis 2015;47(6): 512-517

26 Qazi TM, O’Brien MJ, Farraye FA, Gould RW, Chen CA, Schroy PC III. Epidemiology of goblet cell and microvesicular hyperplastic polyps. Am J Gastroenterol 2014;109(12):1922-1932

27 Kim HY, Kim SM, Seo JH, Park EH, Kim N, Lee DH. Age-specific prevalence of serrated lesions and their subtypes by screening colonoscopy: a retrospective study. BMC Gastroenterol 2014; 14:82-88

28 Szylberg Ł, Janiczek M, Popiel A, Marszałek A. Serrated polyps and their alternative pathway to the colorectal cancer: a systematic review. Gastroenterol Res Pract 2015;2015:573814

29 Yang HM, Mitchell JM, Sepulveda JL, Sepulveda AR. Molecular and histologic considerations in the assessment of serrated polyps. Arch Pathol Lab Med 2015;139(6):730-741

30 Wiland HO IV, Shadrach B, Allende D, et al. Morphologic and molecular characterization of traditional serrated adenomas of the distal colon and rectum. Am J Surg Pathol 2014;38(9): 1290-1297

31 Bettington ML, Chetty R. Traditional serrated adenoma: an update. Hum Pathol 2015;46(7):933-938

32 Kalady MF. Sessile serrated polyps: an important route to colorectal cancer. J Natl Compr Canc Netw 2013;11(12):1585-1594

33 Vasen HFA, Tomlinson I, Castells A. Clinical management of hereditary colorectal cancer syndromes. Nat Rev Gastroenterol Hepatol 2015;12(2):88-97

34 Adolph VR, Bernabe K. Polyps in children. Clin Colon Rectal Surg 2008;21(4):280-285

35 Cichy W, Klincewicz B, Plawski A. Juvenile polyposis syndrome. Arch Med Sci 2014;10(3):570-577

36 van Hattem WA, Langeveld D, de Leng WW, et al. Histologic variations in juvenile polyp phenotype correlate with genetic defect underlying juvenile polyposis. Am J Surg Pathol 2011; 35(4):530-536

37 Ricci MT, Salemme M, Villanacci V, Varesco L. The genetics of inherited predispositions to colorectal polyps: a quick guide for clinicians. Colorectal Dis 2015;17(Suppl 1):3-9

38 Waite KA, Eng C. Protean PTEN: form and function. Am J Hum Genet 2002;70(4):829-844

39 Song MS, Carracedo A, Salmena L, et al. Nuclear PTEN regulates the APC-CDH1 tumor-suppressive complex in a phosphatase-independent manner. Cell 2011;144(2):187-199 